University of Nebraska - Lincoln

DigitalCommons@University of Nebraska - Lincoln

Scattering and bound-state solutions to the wave equation for one electron in the presence of a physical dipole

Gordon A. Gallup

University of Nebraska-Lincoln, ggallup1@unl.edu

Follow this and additional works at: https://digitalcommons.unl.edu/physicsgallup

Part of the Physics Commons

Gallup, Gordon A., "Scattering and bound-state solutions to the wave equation for one electron in the presence of a physical dipole" (2009). Gordon Gallup Publications. 53.

https://digitalcommons.unl.edu/physicsgallup/53

This Article is brought to you for free and open access by the Research Papers in Physics and Astronomy at DigitalCommons@University of Nebraska - Lincoln. It has been accepted for inclusion in Gordon Gallup Publications by an authorized administrator of DigitalCommons@University of Nebraska - Lincoln. 


\title{
Scattering and bound-state solutions to the wave equation for one electron in the presence of a physical dipole
}

\author{
G. A. Gallup* \\ Department of Physics and Astronomy, The University of Nebraska-Lincoln, Lincoln, Nebraska 68588-0111, USA
}

(Received 26 March 2009; published 22 July 2009)

\begin{abstract}
This paper presents a quantum-mechanical analysis of the interaction of one electron with a physical dipole (two physically separated charges). Aspects of threshold laws in the continuous spectrum are treated. In addition there are determinations of energies of some dipole bound states. The bound-state calculations are used as a model for certain high dipole moment molecules, where, together with empirical data, an equivalent dipole length may be determined. These model calculations predict that the bound dipole orbital in high dipole molecule ions should have a node between its valence part and the more remote part where the majority of the charge resides. Some results from the two parts of the treatment are brought together to calculate the asymmetry factor of the photoelectron signal for uracil, measured by Schiedt et al.
\end{abstract}

DOI: 10.1103/PhysRevA.80.012511

PACS number(s): 31.15.ae, 33.60.+q, 34.80.-i

\section{INTRODUCTION}

There has been current interest in electron interactions with supercritical dipole moments, in both positive- and negative-energy regimes. In the positive-energy case the dipole moment can affect the scattering wave function in ways that influence many processes. We shall be particularly interested in the threshold behavior predicted for resonance lifetimes $(\Gamma)$, and, through this, the dissociative electron attachment and vibrational excitation (VE). Experiments measuring the photodetachment energy of dipole bound-state (DBS) systems are also being made currently. In the past most theoretical analyses of dipole problems have relied upon a point-dipole model. This has the disadvantage of modifying the Schrödinger equation's $r^{-2}$ singularity at the origin in such a way that simple formulas for the wave function cannot be used there directly. In this paper, we examine the motion of an electron predicted by solutions of Schrödinger's equation for a potential due to a physical dipole (PD), i.e., equal magnitude positive and negative point charges separated by a finite distance $\rho$. This potential has no difficulties at the origin or at the charges. The electric-dipole moment $D=q_{+} \rho$. Unless stated otherwise, in this paper the orientation of the dipole vector is in the positive $z$ direction.

Schrödinger's equation for an electron is separable in prolate ellipsoidal coordinates for a potential consisting of two fixed charges. This fact was exploited in an early work on the $\mathrm{H}_{2}^{+}$ion and has also been extended to the motion of an electron in the presence of two different nuclei [1-3]. Nonetheless, states for the $\left|q_{-}\right|=q_{+}$charge specification treated here have rarely been discussed. Komorov et al. [4] have a chapter in their book discussing PDs but no numerical results are presented. Numerical studies were made by Takayanagi and Itikawa [5] for scattering from a PD and by Wallis et al. [6] for energies of DBSs.

As stated, Schrödinger's equation in this case is separable in prolate ellipsoidal coordinates, which are discussed in numerous places, see, for example, [7]. Using

\footnotetext{
*ggallup1@unl.edu; http://www.unl.edu/ggallup/
}

$$
\psi(\eta, \xi, \phi)=T(\eta) S(\xi) \Phi(\phi),
$$

the separated equations are

$$
\begin{gathered}
-\lambda T=\frac{d}{d \eta}\left[\left(1-\eta^{2}\right) \frac{d T}{d \eta}\right]-\left(\epsilon \eta^{2}+2 D \eta+\frac{m^{2}}{1-\eta^{2}}\right) T, \\
\lambda S=\frac{d}{d \xi}\left[\left(\xi^{2}-1\right) \frac{d S}{d \xi}\right]+\left(\epsilon \xi^{2}-\frac{m^{2}}{\xi^{2}-1}\right) S,
\end{gathered}
$$

and

$$
m^{2} \Phi=-\frac{d^{2} \Phi}{d \phi^{2}} .
$$

In these equations the new variable, $\epsilon=E \rho^{2} / 2$, has been introduced, where $E$ is the electronic energy, and $D$ is the system dipole moment. Atomic units are used throughout.

Equation (4) is by far the easiest to deal with. In fact, for the calculations we report on, only $m=0$ is an important physical case. Nonzero $m$ values are actually easily treated but no molecule is likely to have a large enough dipole moment to show significant dipole moment effects then.

Equation (2) is also not difficult to treat, expanding $T$ in an infinite series of associated Legendre functions [1-3]. The eigenvalues $\lambda$ can then be obtained numerically as indicated in Ref. [8]. The situation here is similar to that for $m$. Even for $m=0$ only the algebraically lowest $\lambda$ is physically relevant for our purposes.

Equation (3) is, however, more challenging. Except for a different relation between $\lambda=\lambda(\epsilon, D)$ and $\epsilon$, it is identical with the form obtained when the Helmholtz equation is separated in prolate ellipsoidal coordinates, see Meixner [9], Flammer [10], and Komorov et al. [4], and references therein. Some further details on the background to Eq. (3) are in the Appendix. When a singular point analysis is performed on the $\xi$ equation, it is found that there are regular singular points at $\xi= \pm 1$ and an essential singularity at $\xi$ $=\infty$. It is thus a confluent form of Lamé's equation with no known closed solutions in terms of familiar functions. In general we treat it numerically using the Bulirsch-Stör method [11]. Reasons for this decision are outlined in Sec. 
III and the Appendix. We observe here that, however, a series-expansion solution near the $\xi=1$ pole is needed. No difficulty is encountered since only Coulomb singularities are involved.

Concerning the PD Schrödinger's equation, we make one further comment that deals with its behavior as $\rho \rightarrow 0$. As is well known, under these circumstances $\eta \rightarrow \cos (\theta)$ of spherical coordinates, and Eq. (2) passes exactly into the angular equation for a point dipole in spherical coordinates, which at that point is independent of the energy. At the same time, $\rho \xi \rightarrow 2 r$, and $4 \epsilon \rho^{-2} \rightarrow 2 E$. Thus Eq. (3) goes directly into the spherical Bessel equation for a point dipole. We note that, asymptotically, all of the energy dependence devolves to the radial equation.

Before taking up solutions to Schrödinger's equation, in Sec. II we discuss some properties of the PD potential. Section III gives a brief discussion of the solutions of Eq. (3) in general, and in Secs. IV and V we discuss applications to physical problems for the positive- and negative-energy regimes, respectively. One use for the tables in Sec. IV is to compare the PD results to experimental photodetachment threshold behavior.

\section{POTENTIAL OF A PD}

It is of interest to examine the behavior of the PD, comparing it to the more commonly considered point-dipole potential. We do this by computing the ratio of the two potentials. In ellipsoidal coordinates easy calculations give

$$
V_{\text {point }}=\frac{4 D \xi \eta}{\rho^{2}\left(\xi^{2}+\eta^{2}-1\right)^{3 / 2}}
$$

and

$$
V_{\text {physical }}=\frac{4 D \eta}{\rho^{2}\left(\xi^{2}-\eta^{2}\right)}
$$

Therefore,

$$
\begin{gathered}
\quad \frac{V_{\text {physical }}}{V_{\text {point }}}=\frac{\left[1+\left(\eta^{2}-1\right) / \xi^{2}\right]^{3 / 2}}{1-(\eta / \xi)^{2}} \\
=1+\left(5 \eta^{2}-3\right) /\left(2 \xi^{2}\right)+O\left(\xi^{-4}\right), \\
\rightarrow 1+\rho^{2}\left[5 \cos (\theta)^{2}-3\right] /\left(8 r^{2}\right)+O\left((\rho / r)^{4}\right),
\end{gathered}
$$

and the ratio (in ellipsoidal coordinates) is everywhere, independent of both $D$ and $\rho$. This is clearly not the case for $\rho$ in spherical coordinates. By symmetry the PD has no quadrupole moment, and all higher moments are due to its odd multipoles starting with the octopole. In the longitudinal directions, the physical version is the one that is larger in magnitude, whereas transversely, where both are near zero anyway, the point version is the larger. We can obtain an idea of the impact of the octopole term by noting that it has the same $r$ dependence as a polarization potential, and in the $\cos (\theta)$ $= \pm 1$ directions, the magnitude of the $(\rho / r)^{4}$ term is equivalent to a polarization potential with $\alpha=\rho^{3} / 2$, if $|D / \rho| \approx|e|$.

\section{III. $\xi$ EQUATION FOR $\boldsymbol{m}=0$}

We rewrite Eq. (3) (with $m=0$ ) in the form

$$
\begin{aligned}
& {[(\xi-1)+2] S^{\prime \prime}+\left[2+\frac{2}{\xi-1}\right] S^{\prime}+\left[\epsilon(\xi-1)+2 \epsilon+\frac{\epsilon-\lambda}{\xi-1}\right] S} \\
& \quad=0
\end{aligned}
$$

and substituting a power-series expansion about the point $\xi$ $=1$,

$$
S(\xi)=\sum_{k} a_{k}(\xi-1)^{k+s},
$$

we obtain a recursion relation for the coefficients, $a_{k}$, where $k=0,1,2, \ldots$, with four terms, in general, and the first two having only two and three terms. The indicial equation has the double root, $s=0$, and the two-term relation,

$$
a_{1}=-\frac{1}{2}(\epsilon-\lambda) a_{0}
$$

provides the one solution we need, analytical at $\xi=1$. Standard methods for analyzing differential equations [12] show that the other solution is logarithmic at $\xi=1$. In general we have

$$
a_{k}=-\frac{1}{2 k^{2}}\left\{[\epsilon-\lambda+k(k-1)] a_{k-1}+2 \epsilon a_{k-2}+\epsilon a_{k-3}\right\} .
$$

This may be simplified using further variable changes but the present form gives a convergent series in the range $|\xi-1|$ $<2$, which is large enough for our needs.

For positive energies Meixner [9] has given an analytical solution of Eq. (3) as an infinite series of generalized spherical Bessel functions. An outline of this approach is given in the Appendix. A treatment using the Meixner form would yield only tables of numbers for phase shifts and threshold values since all of the series must be evaluated numerically. The same result is true if we use the Bulirsch-Stör method to integrate a solution outward, starting from the analytic solution implied by Eq. (13). It is our judgment that the latter is an easier approach. Further details concerning this decision occur below and in the Appendix.

For negative energies, series solution expressions for Eq. (3) are not so difficult. Nevertheless, the Bulirsch-Stör method may again be used to match inwardly integrated solutions to series solutions in the vicinity of the $\xi=1$ point.

\section{POSITIVE ENERGIES}

Takayanagi and Itikawa [5] have calculated scattering cross sections for the PD potential in ranges of energies and dipole moments. They, however, do not deal explicitly with the threshold behavior, which is the focus of our interest.

\section{A. Threshold behavior}

A standard analysis of Eq. (3) shows that the solution with $S(1)=a_{0}$ has the asymptotic behavior,

$$
S(\xi) \rightarrow C \frac{\sin (\sqrt{\epsilon} \xi+\delta)}{\sqrt{\xi^{2}-1}}
$$




$$
=C^{\prime} \frac{\sin (K r+\delta)}{\sqrt{k} r},
$$

where $C$ and $C^{\prime}$ are constants. The $a_{0}$ [see Eq. (11)] constant in our solution is still free; therefore, if $C^{\prime}$ is the result in Eq. (15) when $a_{0}=1$, setting $a_{0}=1 / C^{\prime}$ will yield the solution going to an energy normalized plane wave. This analysis follows that of O'Malley [13], where, in his Eq. (3), our $a_{0}$ plays the role of his $N_{l}(k)$. Thus, when investigating threshold effects in photodetachment photoelectron (PDPE) spectroscopy, VE, and resonance lifetimes $(\Gamma)$, the physical dipole moment $(\mathrm{PDM})$ predicts they would be proportional to $\left|a_{0}\right|^{2}$.

As stated in Sec. III we use the Bulirsch-Stör [11] technique to integrate Eq. (3). This equation has very smooth coefficients and no difficult points in the region $\xi>1$. Starting with values and slopes determined with Eqs. (11) and (13), we have successfully carried out, with complete stability, integrations up to $\xi \approx 10^{5}$, which is around $8 \times 10^{-6} \mathrm{~m}$ for $\rho=1.5 \AA$. Technically, carrying a numerical integration out to a finite point and then matching the result to Eq. (15) is equivalent to breaking off the potential function at that point, turning it into what is really a short-range potential. The distance above is, however, deemed large enough to ignore this effect for our purposes [14].

As the outward integration is continued, after a threshold distance is reached, values of $C^{\prime}$ and the phase shift are determined approximately in every wavelength, and four (third degree) or six (fifth degree) of these values were extrapolated to $1 / \xi=0$. Standard polynomial extrapolation techniques were used [15], and the differences between the third or fifth degree polynomial versions were insignificant. The values so calculated are subjected to a number of analyses in the next sections.

\section{B. Free particle}

As a test of our procedure, we calculate the phase shift and threshold behavior for the ellipsoidal representation of the solution of the Helmholtz equation, a plane wave. This is easily done by setting $D=0$. We use

$$
a_{0}=E^{a}\left(b+c E+d E^{2}\right)
$$

to represent the threshold behavior. Figure 1 shows a graph of this function for our calculated points. Using a standard least square criterion, the values we arrived at were $a$ $=0.25000, b=0.52068, c=-0.00427$, and $d=0.00004$. The phase shift is -0.000001 . As far as $a$ and the phase shift are concerned, this is considered satisfactory agreement with the known exact values for the energy normalized plane wave.

\section{Phase shifts and threshold behavior as a function of $D$}

In both Eqs. (2) and (3) $E$ and $\rho$ appear only in the combination $\epsilon=\rho^{2} E / 2=(\rho k / 2)^{2}$. Therefore, in giving a survey of results for different $D$ values, it is useful to use the dimensionless $\kappa=\rho k / 2=\sqrt{\epsilon}$ in giving results.

We have fitted two formulas to the threshold data that are determined numerically. Both to satisfy various theoretical

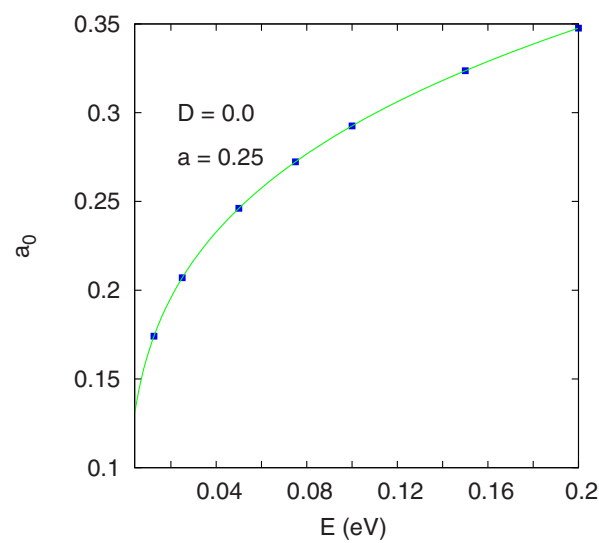

FIG. 1. (Color online) $D=0.0 D$. The $a_{0}$ values as a function of energy. The value of the $a$ exponent in Eq. (16) is shown in the graph.

results and to obtain the best fits by a least square criterion, one formula works better for moments in the point-dipole subcritical range and the other for moments in the pointdipole supercritical range. The subcritical form is

$$
a_{0}=a \kappa^{x}\left(1+b \kappa^{2}\right) .
$$

For supercritical cases we adapt a form given by Fabrikant $[16,17]$

$$
a_{0}=\frac{1}{a+b \cos [2 c \ln (\kappa)+d]},
$$

where, in this case, the $c$ parameter is to be optimized in the fitting procedure. This is done in an attempt to compensate for the variability of $\lambda$ since, in these equations, $\lambda=\lambda(\kappa)$ and is energy dependent.

Table I gives the results for the coefficients in Eq. (17) for the subcritical range, and Table II provides the same for Eq. (18) in the other range. The phase shifts have been collected for these calculations, and are presented both in Table III and as a pseudo-three-dimensional graph in Fig. 2. From their calculations, Takayanagi and Itikawa [5] give a graph of the phase shift as a function of $D$ for two energies. Qualitatively, their curves are completely consistent with Fig. 2.

The entries in Tables I and II are suitable for interpolation for other dipole moments. Because of its greater interest, this has been done for the supercritical case to produce a pseudo-

TABLE I. Parameters for subcritical fits. See Eq. (17).

\begin{tabular}{lccc}
\hline \hline \multicolumn{1}{c}{$D$} & $a$ & $x$ & $b$ \\
\hline $0.25^{\mathrm{a}}$ & 0.84012 & 0.49352 & -0.04488 \\
0.50 & 0.83982 & 0.47380 & -0.03613 \\
0.75 & 0.83673 & 0.43988 & -0.00721 \\
1.00 & 0.82620 & 0.38983 & 0.07240 \\
1.25 & 0.80013 & 0.32001 & 0.26619 \\
1.50 & 0.74529 & 0.22376 & 0.69806 \\
1.62389 & 0.81129 & 0.17796 & 1.09624 \\
\hline \hline
\end{tabular}

${ }^{\mathrm{a}}$ Debye. 
TABLE II. Parameters for supercritical fits. See Eq. (18).

\begin{tabular}{|c|c|c|c|c|c|c|c|}
\hline $\begin{array}{c}D \\
\text { (a.u.) }\end{array}$ & $a$ & $b$ & $c$ & $d$ & $\Sigma^{a}$ & Amp. ${ }^{b}$ & $\sqrt{|\lambda+1 / 4|}$ \\
\hline 0.7 & 0.93442 & 1.25271 & 0.13393 & 1.40636 & $8.699 e-03$ & 0.50219 & 0.21303 \\
\hline 0.75 & 0.87407 & 0.83232 & 0.18837 & 1.25383 & $9.445 e-03$ & 0.40037 & 0.29137 \\
\hline 0.8 & 0.99414 & 0.49319 & 0.26820 & 1.29025 & $9.243 e-03$ & 0.32757 & 0.35525 \\
\hline 0.85 & 1.05239 & 0.30730 & 0.38376 & 1.46937 & $1.386 e-02$ & 0.27465 & 0.41134 \\
\hline 0.9 & 1.03035 & 0.23666 & 0.48631 & 1.61464 & $1.620 e-02$ & 0.23398 & 0.46235 \\
\hline 0.95 & 1.00443 & 0.19493 & 0.55311 & 1.56394 & $1.155 e-02$ & 0.20162 & 0.50973 \\
\hline 1.0 & 0.98588 & 0.16401 & 0.60814 & 1.46454 & $1.062 e-02$ & 0.17526 & 0.55433 \\
\hline 1.1 & 0.97406 & 0.12825 & 0.67155 & 1.01760 & $1.020 e-02$ & 0.13508 & 0.63721 \\
\hline 1.2 & 0.96821 & 0.10357 & 0.72504 & 0.63462 & $1.050 e-02$ & 0.10622 & 0.71374 \\
\hline 1.3 & 0.96205 & 0.08215 & 0.78544 & 0.34899 & $1.421 e-02$ & 0.08978 & 0.78546 \\
\hline 1.4 & 0.95745 & 0.06588 & 0.84898 & 0.10428 & $4.068 e-02$ & 0.06850 & 0.85332 \\
\hline 1.5 & 0.95473 & 0.05367 & 0.91290 & -0.10766 & $3.055 e-02$ & 0.05592 & 0.91797 \\
\hline 1.6 & 0.95338 & 0.04415 & 0.97988 & -0.27209 & $1.152 e-02$ & 0.04604 & 0.97987 \\
\hline 1.7 & 0.95234 & 0.03638 & 1.03991 & -0.46058 & $4.666 e-03$ & 0.03818 & 1.03939 \\
\hline 1.8 & 0.95156 & 0.03028 & 1.09754 & -0.65150 & $1.319 e-03$ & 0.03188 & 1.09681 \\
\hline 1.9 & 0.95105 & 0.02546 & 1.15262 & -0.83972 & $1.063 e-03$ & 0.02677 & 1.15234 \\
\hline 2.0 & 0.95073 & 0.02145 & 1.20573 & -1.02719 & $1.038 e-03$ & 0.02261 & 1.20617 \\
\hline 2.1 & 0.95050 & 0.01820 & 1.25875 & -1.20609 & $9.204 e-04$ & 0.01918 & 1.25846 \\
\hline 2.2 & 0.95034 & 0.01557 & 1.31004 & -1.38346 & $1.298 e-03$ & 0.01635 & 1.30933 \\
\hline 2.3 & 0.95021 & 0.01331 & 1.35951 & -1.56172 & $9.438 e-04$ & 0.01399 & 1.35890 \\
\hline 2.4 & 0.95014 & 0.01145 & 1.40784 & -1.74021 & $1.660 e-03$ & 0.01202 & 1.40726 \\
\hline 2.5 & 0.95006 & 0.00986 & 1.45548 & -1.91368 & $8.990 e-04$ & 0.01036 & 1.45451 \\
\hline 2.6 & 0.95004 & 0.00857 & 1.50203 & -2.08278 & $3.671 e-03$ & 0.00896 & 1.50072 \\
\hline
\end{tabular}

\footnotetext{
${ }^{\mathrm{a}}$ Average relative standard deviation of fit.

${ }^{\mathrm{b}}$ See text.

${ }^{\mathrm{c}}$ Point dipole value.
}

three-dimensional graph of $a_{0}$ values in Fig. 3 .

Some trends in the numbers in Table II may be commented upon. The constant term in the denominator is not far from one over the range of values. The amplitude of the logarithmic oscillations falls rapidly with increasing $D$. In the last two columns of Table II we give the point-dipole predictions (see Ref. [17]) for the amplitude of the oscillations and the imaginary part of the exponents of $r$ in spherical coordinates. These may be compared to the fitted values of $c$ in the third and fourth columns. For the range of $D$ values calculated, the $c$ values are smaller but the difference appears to be decreasing as $D$ becomes larger. This is reasonable since the energy term in Eq. (2) will become relatively less important as $D$ becomes larger. Nevertheless, these values from the PDM are qualitatively very similar to those from point-dipole analyses. The differences can be attributed to the octopole and higher multipoles present in the physical dipole potential, see Sec. II.

\section{Passage from spheroidal to spherical coordinates at zero energy}

An examination of Eq. (13), the recursion relation, shows that it is satisfactory for all finite values of $E$ and $\rho$. For simplicity, let us consider the $E=0$ case. Equation (3) becomes

$$
\left(\xi^{2}-1\right) S^{\prime \prime}+2 \xi S^{\prime}-\lambda S=0 .
$$

At the same time, the $\rho=0$ (holding $D$ fixed) Schrödinger's equation is

$$
r^{2} S^{\prime \prime}+2 r S^{\prime}-\lambda S=0 .
$$

The value of $\lambda$ is the same in Eqs. (19) and (20).

We modify Eq. (19) to

$$
\left(\xi^{2}-\delta^{2}\right) S^{\prime \prime}+2 \xi S^{\prime}-\lambda S=0,
$$

which is equivalent to

$$
\left[(\xi-\delta)^{2}+2 \delta(\xi-\delta)\right] S^{\prime \prime}+2[(\xi-\delta)+\delta] S^{\prime}-\lambda S=0,
$$

and examine the behavior of the solution as $\delta \rightarrow 0, \delta$ being real and positive. Inserting the power-series solution

$$
S(\xi)=\sum_{k} a_{k}(\xi-\delta)^{k+s},
$$

we obtain the indicial equation, 
TABLE III. Table of phase shifts for a range of dipole moments and $\kappa$.

\begin{tabular}{|c|c|c|c|c|c|c|c|c|c|c|c|}
\hline \multicolumn{12}{|c|}{ Phase shifts } \\
\hline$D / \kappa$ & 0.01 & 0.03 & 0.05 & 0.07 & 0.09 & 0.11 & 0.13 & 0.15 & 0.17 & 0.19 & 0.21 \\
\hline 0.0 & 0.0 & 0.0 & 0.0 & 0.0 & 0.0 & 0.0 & 0.0 & 0.0 & 0.0 & 0.0 & 0.0 \\
\hline 0.1 & 0.01038181 & 0.01015504 & 0.00992836 & 0.00970707 & 0.00947455 & 0.00923156 & 0.00902526 & 0.00880258 & 0.00858155 & 0.00843558 & 0.00824576 \\
\hline 0.2 & 0.04207301 & 0.04102542 & 0.04001947 & 0.03904897 & 0.03808742 & 0.03714023 & 0.03624566 & 0.03528308 & 0.03437164 & 0.03337850 & 0.03270279 \\
\hline 0.3 & 0.09667498 & 0.09382386 & 0.09121982 & 0.08874518 & 0.08643018 & 0.08408750 & 0.08177587 & 0.07971880 & 0.07748294 & 0.07536929 & 0.07313627 \\
\hline 0.4 & 0.17777023 & 0.17108020 & 0.16542490 & 0.16030931 & 0.15548922 & 0.15090703 & 0.14660423 & 0.14227340 & 0.13802368 & 0.13434526 & 0.13009522 \\
\hline 0.5 & 0.29253451 & 0.27746658 & 0.26599417 & 0.25604299 & 0.24709693 & 0.23892921 & 0.23118704 & 0.22368885 & 0.21684679 & 0.20991170 & 0.20392353 \\
\hline 0.6 & 0.45618410 & 0.42124032 & 0.39835621 & 0.37991313 & 0.36421063 & 0.35011598 & 0.33724518 & 0.32552071 & 0.31412433 & 0.30383680 & 0.293 \\
\hline 0.7 & 0.70317417 & 0.61732364 & 0.57121276 & 0.53772815 & 0.51037659 & 0.48706532 & 0.46667324 & 0.44808626 & 0.43039572 & 0.41483539 & 0.40036869 \\
\hline 0.8 & 1.11570843 & 0.89018696 & 0.79693293 & 0.73613588 & 0.69026511 & 0.65294691 & 0.62038799 & 0.59283501 & 0.56684066 & 0.54421014 & 0.52348910 \\
\hline 0.9 & 1.81369550 & 1.27108779 & 1.08970553 & 0.98325401 & 0.90798091 & 0.84911513 & 0.80083422 & 0.75996015 & 0.72381617 & 0.69099030 & 0.66160102 \\
\hline 1.0 & 2.58781598 & 1.75913899 & 1.45183094 & 1.28016612 & 1.16408700 & 1.07703137 & 1.00705106 & 0.94990462 & 0.89955173 & 0.85571725 & 0.81582739 \\
\hline 1.1 & 3.12056426 & 2.26549949 & 1.85544820 & 1.61404030 & 1.45156937 & 1.33063026 & 1.23530373 & 1.15788893 & 1.09189551 & 1.03463373 & 0.98320887 \\
\hline 1.2 & 3.50290765 & 2.69450983 & 2.24757476 & 1.95683518 & 1.75265429 & 1.59929888 & 1.47849011 & 1.37901692 & 1.29640792 & 1.22466146 & 1.16075383 \\
\hline 1.3 & 3.83735405 & 3.03942222 & 2.59218153 & 2.27980116 & 2.04805693 & 1.86832417 & 1.72598080 & 1.60636108 & 1.50719477 & 1.42201597 & 1.34464851 \\
\hline 1.4 & 4.16970125 & 3.33128090 & 2.88778836 & 2.56903867 & 2.32327411 & 2.12776354 & 1.96736054 & 1.83326642 & 1.71712727 & 1.61796400 & 1.53077921 \\
\hline 1.5 & 4.51703155 & 3.59716034 & 3.14780862 & 2.82676119 & 2.57390406 & 2.36791202 & 2.19516811 & 2.04984710 & 1.92389298 & 1.81275732 & 1.71539118 \\
\hline 1.6 & 4.87914407 & 3.85222994 & 3.38559120 & 3.05977214 & 2.80231539 & 2.59092877 & 2.41149952 & 2.25513228 & 2.12141822 & 2.00144140 & 1.89562439 \\
\hline 1.7 & 5.24433843 & 4.10598369 & 3.61199374 & 3.27578230 & 3.01341212 & 2.79556164 & 2.61223907 & 2.45063717 & 2.30931507 & 2.18376588 & 2.06973461 \\
\hline 1.8 & 5.59829476 & 4.36196446 & 3.83275511 & 3.48246923 & 3.21211399 & 2.98977610 & 2.79989271 & 2.63304749 & 2.48770340 & 2.35672347 & 2.23700516 \\
\hline 1.9 & 5.93279961 & 4.62000601 & 4.05024666 & 3.68145533 & 3.40244790 & 3.17372986 & 2.97771808 & 2.80859214 & 2.65753783 & 2.52199238 & 2.39749011 \\
\hline 2.0 & 6.24766328 & 4.87846299 & 4.26811962 & 3.87891933 & 3.58736666 & 3.34993673 & 3.15156784 & 2.97685313 & 2.82011959 & 2.68044831 & 2.55171699 \\
\hline
\end{tabular}

$$
\delta s^{2}=0
$$

and the two-term recursion relation

$$
2 \delta a_{k+1}=\frac{k(k+1)-\lambda}{(k+1)^{2}} a_{k}
$$

and the series will converge while $|\xi-\delta|<2 \delta$. These relations hold so long as $\delta>0$. When $\delta$ becomes 0, Eq. (24) no longer determines $s$, and the recursion formula degenerates into one term, giving the two solutions

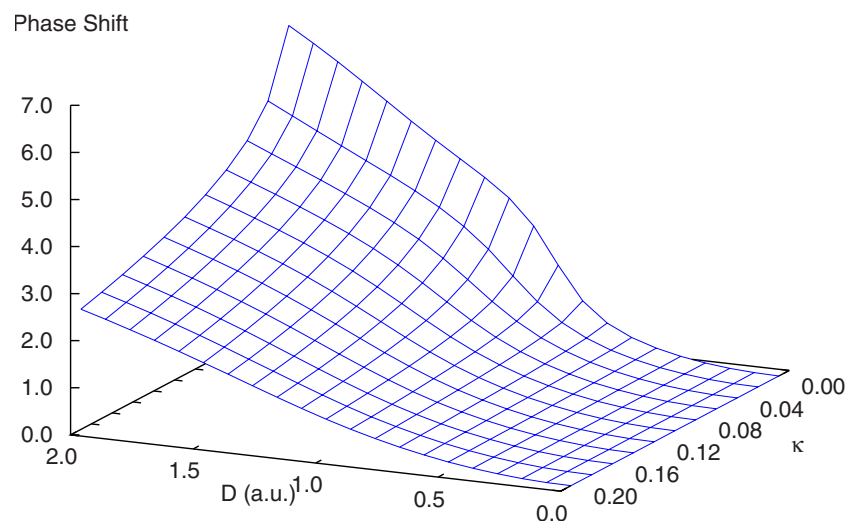

FIG. 2. (Color online) "Three-dimensional" plot of the phase shift versus $D$ and $\kappa$. Numerical values are in Table III.

$$
S=r^{-1 / 2 \pm \sqrt{1 / 4+\lambda}} .
$$

Although the circle of convergence becomes smaller as $\delta$ $\rightarrow 0$, the determination of $s$ changes discontinuously with the appearance of the $1 / r^{2}$ singularity. At that point the characteristic possibly complex powers of $r$ appear.

\section{NEGATIVE ENERGIES}

Apparently, only Wallis et al. [6] have studied the DBS energies of the PD model, giving tabular presentations of DBS energies. They used the analytical approach of Baber and Hasse [2], and give energies for a number of states assuming two $|e|$ magnitude charges at various distances. Appropriate scaling can, in principle, provide for other physical

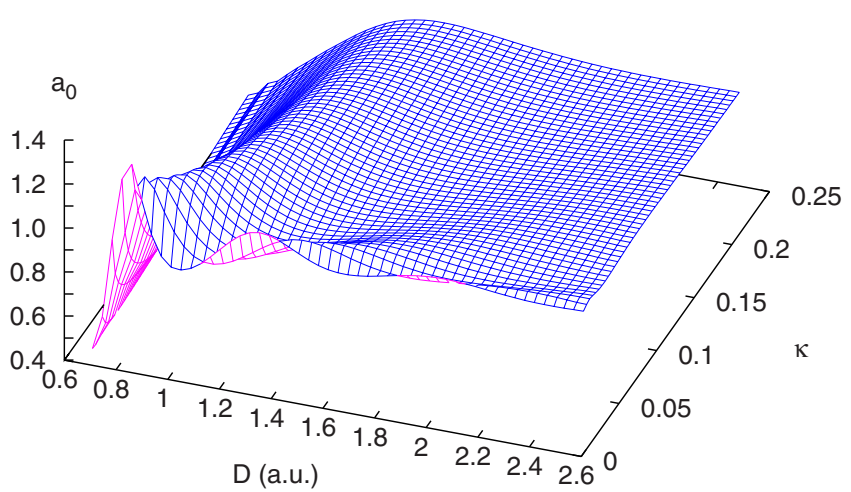

FIG. 3. (Color online) Three-dimensional plot of $a_{0}$ versus $D$ and $\kappa$. Interpolated values from Table II. 
TABLE IV. The EPDL for the four pyrimidine bases. The column headed " $q / e$ " is the equivalent charge of the dipole in electrons.

\begin{tabular}{|c|c|c|c|c|c|c|}
\hline & \multirow{2}{*}{$\begin{array}{c}\mathrm{DE} \\
(\mathrm{eV})\end{array}$} & \multicolumn{2}{|c|}{$D($ debye $)$} & \multirow{2}{*}{$\begin{array}{c}\text { EPDL } \\
(\AA)\end{array}$} & \multirow[b]{2}{*}{$q / e$} & \multirow[b]{2}{*}{$\rho_{a}$} \\
\hline & & Theor. $^{\mathrm{a}}$ & Exp. $^{c}$ & & & \\
\hline Uracil $^{\mathrm{b}}$ & 0.093 & 4.4295 & 4.7 & 0.1992 & 4.633 & 0.0301 \\
\hline Thymine $^{\mathrm{b}}$ & 0.069 & 4.3929 & 4.6 & 0.2197 & 4.165 & 0.0262 \\
\hline 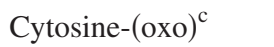 & 0.230 & 6.5021 & $6.6-8$ & 0.6712 & 2.018 & 0.0442 \\
\hline Cytosine-(hydroxo) $^{\mathrm{c}}$ & 0.085 & 4.7260 & $6.5-8$ & 0.2911 & 3.382 & 0.0321 \\
\hline
\end{tabular}

situations. Nonetheless, we repeat their calculations because we require values from difficult to interpolate regions of their tables.

\section{A. Fitting solutions of the model potential to experiment}

One independent parametrization of the energies of DBSs depends upon the dipole moment, the dipole length, and the quantum number. In line with the normal convention of Sturm-Liouville theory, we take the quantum numbers $n$ $=0,1, \ldots$ to be the number of nodes in the solution interior to the boundaries. In general, with the dipole potential, one finds the formula,

$$
E_{n}=E_{0} \exp (-a n),
$$

to give a reasonably accurate representation of the energies. $E_{0}$ and $a$ in Eq. (27) are parameters that depend upon the model. When we wish to fit our model to a molecule with a known dipole moment and a known DBS energy, we find that the solution is not unique because both the dipole length and the quantum number are unknown. There are several dipole length- $n$ pairs that can be obtained. We will find that, however, only one of these pairs will be physically reasonable. When the dipole moment of a molecule is calculated using the dipole operator, the value obtained is that seen from an asymptotic distance. Thus the value is the distance between the centroids of positive and negative charges times the sum of the atomic numbers of the atoms, or, alternatively, using the atomic number total $\sum_{i} Z_{i}$, the "asymptotic" dipole length is $\rho_{a}=D / \Sigma_{i} Z_{i}$. Therefore, when we do our fitting, we should look for an empirical $\rho$ that is not shorter than $\rho_{a}$ but also not substantially larger than the molecule. There is also a computational criterion. Direct calculations of the DBS orbital in molecules show that it is likely to correspond to the $n=1$ state of the model. For the cases we examine this gives us a unique solution.

With the molecules we are treating there is near coincidence between the centroid of positive charge and the centroid of mass because the principle isotopes of $\mathrm{C}, \mathrm{N}$, and $\mathrm{O}$ have a mass number twice their atomic number. Only $\mathrm{H}$ is different, and, indeed, would be the same for the deuterated versions. Thus, we expect the asymptotic PD to be located in the molecule essentially on top of the mass centroid. The results using these criteria and this point of view are given in the next section.

\section{B. Comparisons to experiment}

There has been experimental and theoretical interest in some of the pyrimidine bases comprising the DNA codes. Hendricks et al. [18] have measured the PDPE spectra of the dipole bound states (DBSs) of uracil and thymine. More recently, Schiedt et al. [19] have remeasured uracil and thymine, and have given new results for two isomers of cytosine. The experimental detachment energies are in Table IV.

Our calculations again use the numerical approach for negative-energy states, and the Bulirsch-Stör approach easily provides solutions by connecting inward integrations with the analytic inverse log derivative obtained with Eqs. (11) and (13), thereby determining the energy. In this section we compare our calculated DBS binding energies with experiment for the pyrimidine bases uracil, thymine, and two isomers of cytosine. This allows us to determine a semiempirical equivalent physical dipole length (EPDL) to associate with each base. These and the associated wave functions yield important physical information about the nature of the DBS.

In Sec. V C we examine in more detail the results for thymine as an example. The structures and dipole moments were calculated at the $6-311 \mathrm{G}(\mathrm{spd}) \mathrm{MP} 2$ level using the GAMESS computation package [20]. Figure 4 shows graphically the frameworks and dipole moments resulting from these calculations.

Our PD potential will not match the actual potential in the close regions of molecules in general, particularly larger molecules such as those under discussion. It does, however, provide a model potential that is physically reasonable in having, as is true for a real molecule, no parts more singular than a Coulomb singularity. As such, it will allow us to calculate from experimental results an EPDL for each of these molecules. These are presented in Table IV along with the experimental binding energies, experimental and calculated dipole moments, the equivalent charge at the positive end of the dipole, and that headed $\rho_{a}$ is the asymptotic dipole length given in Sec. V A.

The four bases fall roughly into two categories, the oxocytosine being in one and the remainder in the other. Wallis et al. [6] have already pointed out that a DBS for a given dipole moment will be more stable the shorter the PD. Because of the scaling laws of Eqs. (2) and (3), we see that the PD moment predicts that the oxoisomer would have a stabil- 


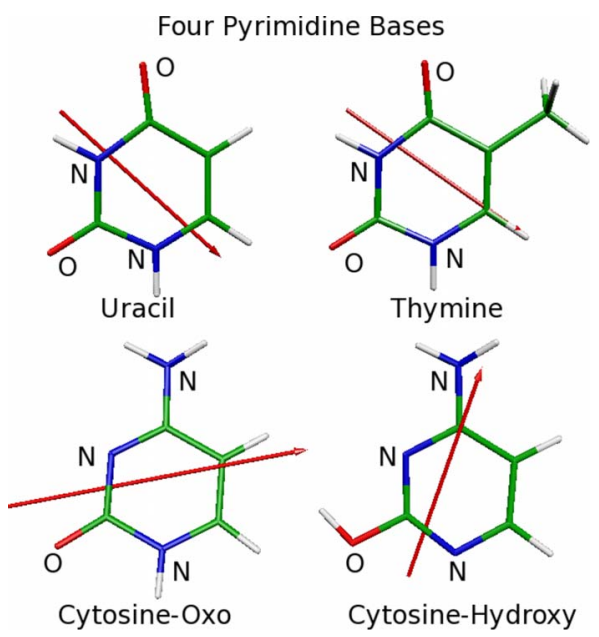

FIG. 4. (Color online) Pyrimidine base frameworks showing dipole vectors. The head of the arrow represents the positive end of the dipole moment. The stick figures are coded to represent different atoms as $\mathrm{H}$ : white, $\mathrm{C}$ : green, $\mathrm{N}$ : blue, and $\mathrm{O}$ : red. Atoms other than $\mathrm{C}$ and $\mathrm{H}$, which should be obvious, are explicitly marked with $\mathrm{N}$ or $\mathrm{O}$.

ity around $1.223 \mathrm{eV}$ if its EPDL were the same length as that of the hydroxy isomer.

In these molecules the $\mathrm{C}=\mathrm{O}$ group is one of the main contributors to the total dipole moment, and Fig. 4 shows that the negative end of the dipole moment is closer to the $\mathrm{C}-\mathrm{O}$ portion of the molecule for cytosine isomers than in the others, where the moment is roughly half way between the two $\mathrm{C}=\mathrm{O}$ bonds.

\section{Nature of the DBS orbital in thymine}

In this section we give for thymine a detailed analysis of the manner in which our PD model represents the DBS orbital. Many workers including the present author $[21,22]$

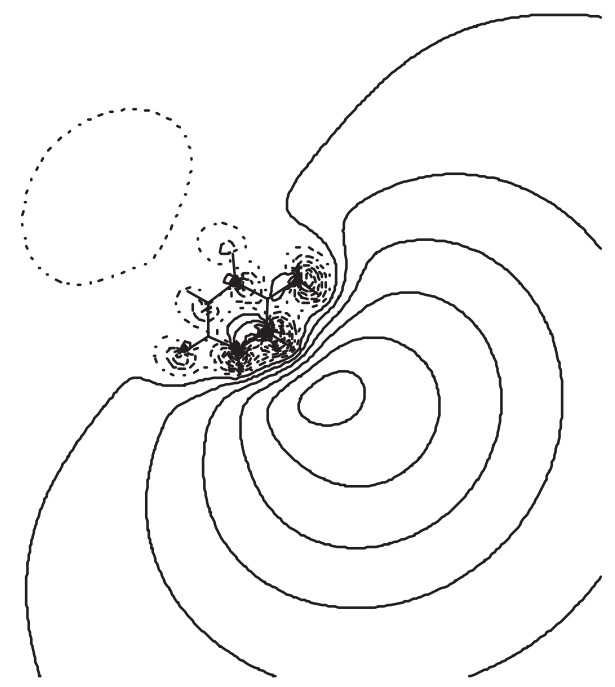

FIG. 5. An extended view of the DBS orbital in thymine. The solid contours indicate positive amplitude and the dashed contours indicate negative amplitude.

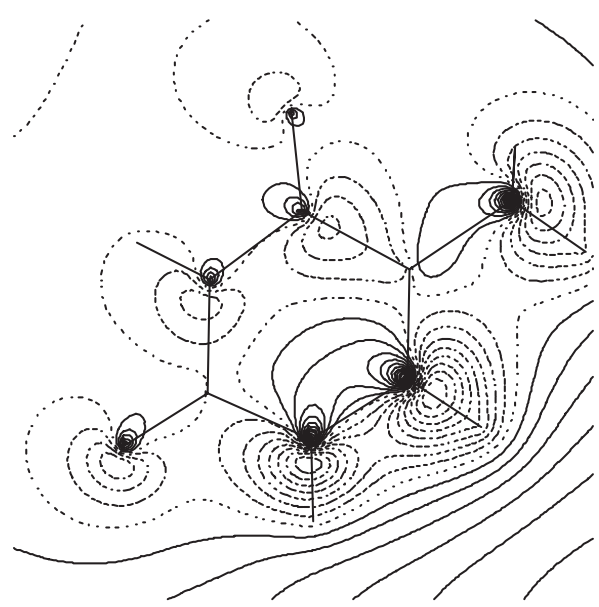

FIG. 6. The valence region of the DBS orbital in thymine. The solid contours indicate positive amplitude and the dashed contours indicate negative amplitude.

have made numerous calculations using quantum chemical computer programs to study DBSs. Our studies [22] also used GAMESS, and we repeat the DBS orbital contour map in Figs. 5 and 6, which show both the extended region and the valence region. The contours of positive amplitude are solid lines and those of negative amplitude are dashed. Perhaps the most noteworthy detail of this orbital function is the obvious node (more obvious in Fig. 6) between its valence part and its extended part. In its simplest approximation, one expects the DBS function to be a linear combination between valence virtual orbital (VOs) and the Gaussians representing the extended part, and there appears no reason the bonding rather than the antibonding combination should not be the lower energy. Nor is it obvious that orbital orthogonality should require this node. In the next few paragraphs we give an argument, based upon the PD model, which suggests that this node is required by the physical and geometric constraints of the system, the relatively large dipole moment, the relatively small size of the system, and the small binding energy.

Table V shows details of these calculations, the first three rows of which are the first three PDPEs for thymine's moment and EPDL given in Table IV. It is clear that only the first-excited DBS of the PD model can provide a close representation of the physical situation.

The last line of Table $\mathrm{V}$ shows the required dipole length if the PD model is to reproduce the experimental PDPE energy for a nodeless radial function. The length is over 200

TABLE V. A table giving various combinations of PD model parameters and detachment energies related to thymine.

\begin{tabular}{ccc}
\hline \hline$n$ & $\begin{array}{c}\text { PDPE } \\
(\mathrm{eV})\end{array}$ & $\begin{array}{c}\text { EPDL } \\
(\AA)\end{array}$ \\
\hline 2 & -0.00014861 & 0.2197 \\
1 & $-0.069^{\mathrm{a}}$ & 0.2197 \\
0 & -26.6486 & 0.2197 \\
0 & $-0.069^{\mathrm{a}}$ & 4.418 \\
\hline \hline
\end{tabular}

${ }^{\mathrm{a}}$ Experimental. 


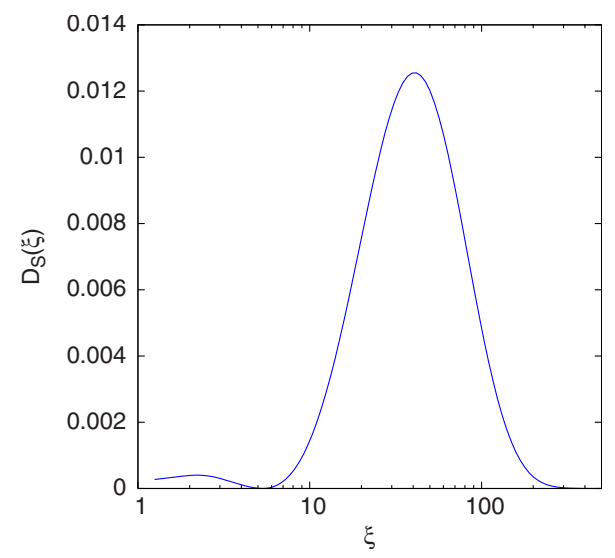

FIG. 7. (Color online) A semilog plot of $D_{S}(\xi)$ [see Eq. (28)] for the first-excited DBS from the PD model of thymine. See line 2 of Table V.

times the $\rho_{a}$ length, and is roughly the size of the molecule. In addition, the PD model itself is, in this case, essentially an exotic H-like atom of nuclear charge of $0.391|e|$ with an embedded $-0.391|e|$ charge at some distance. Such a structure is clearly unrelated physically to molecular thymine. We conclude that only the DBS with one node can produce, with the PD model, the large moment, the relatively small valence region where the moment is produced, and the small PDPE energy. It appears that exchange has relatively little influence on the DBS orbital.

This result is perhaps in one way surprising. One generally finds that the lowest energy state of a potential has no nodes. The complexities of these large molecule DBSs appear to cause one to find an exception to expectations. In any event the PDM provides an explanation for the apparent anomalous behavior.

We add some other information about the model DBS orbitals by giving graphs of the ellipsoidal analog to the spherical radial distribution function. This is obtained with

$$
D_{S}(\xi)=\left(\xi^{2}-\left\langle T\left|\eta^{2}\right| T\right\rangle\right) S(\xi)^{2},
$$

where the expectation value of $\eta^{2}$ is 0.494219 for the thymine case. Figure 7 shows $D_{S}(\xi)$ for the physically appropriate

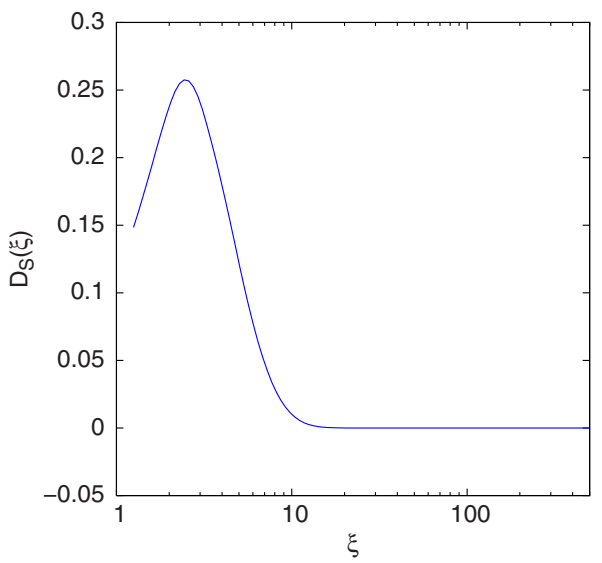

FIG. 8. (Color online) A semilog plot of $D_{S}(\xi)$ [see Eq. (28)] for the ground DBS from the PD model of thymine. See line 3 of Table $\mathrm{V}$.

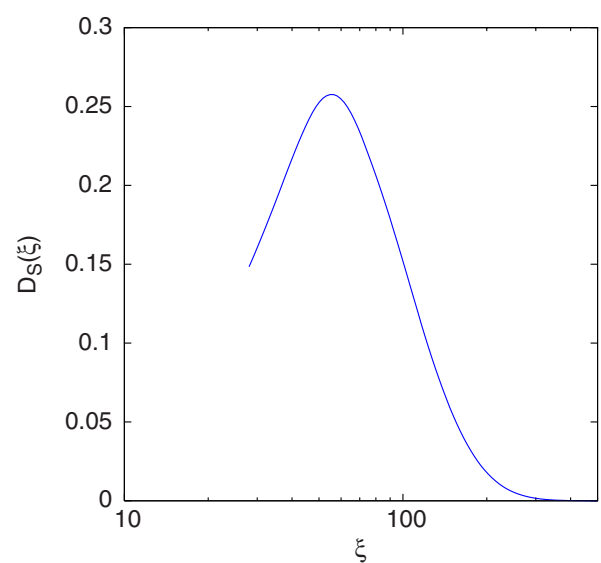

FIG. 9. (Color online) A semilog plot of $D_{S}(\xi)$ [see Eq. (28)] for the ground DBS from the large EPDL PD model of thymine. See line 4 of Table V.

DBS.

In Figs. 8 and 9 we show semilog graphs corresponding to lines 3 and 4 of Table V. These are surprisingly similar only because plotting versus $\xi$ hides the extreme difference between the $\rho$ values in the two cases.

We have also calculated the next-higher state of our PD model (line 1 of Table V). The $S(\xi)$ eigenfunctions both for it and for the physical state are shown together in Fig. 10. If a nonrotating thymine actually had a state with energy near this, it would almost certainly be quenched by rotations at normal temperatures.

Finally, we note that the three energies for thymine that we have obtained are accurately given by Eq. (27), where $E_{0}=-26.6486(\mathrm{eV}), a=5.95639$.

\section{Asymmetry parameter for uracil}

Schiedt et al. [19] also reported on the photoelectric asymmetry parameter for uracil at the optical wavelength of

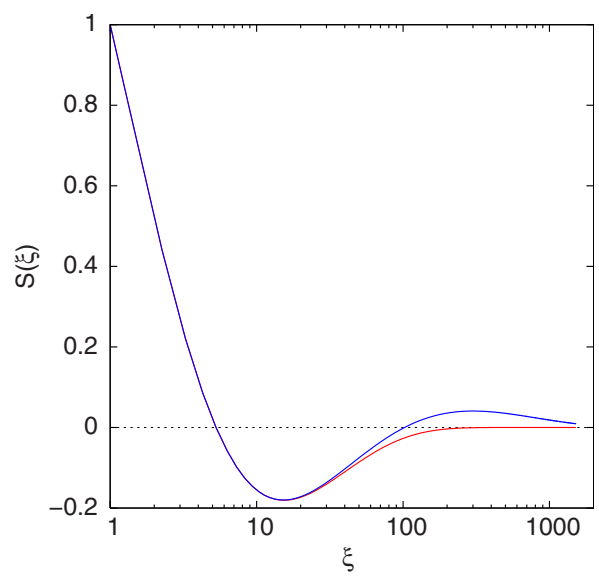

FIG. 10. (Color online) The second and third eigenfunctions for the EPDL parameters corresponding to thymine. (See lines 2 and 3 of Table V.) It is seen that the two functions are virtually indistinguishable out to $\xi \approx 30$ since, inside this region, the potential dominates the energy. 
$1064 \mathrm{~nm}$. The corresponding electron kinetic energy is 1.027 $\mathrm{eV}$, which, with the value of $\rho$ (EPDL) from Table IV, gives $\epsilon=0.0387$. As stated in Sec. I, the equation for the $\eta$ function is

$$
T^{m}(\eta)=\sum_{l} d_{l}^{m} N_{l m} P_{l m}(\eta),
$$

and the values of $d_{l}^{0}$ are $0.8072,-0.5708$, and 0.1490 for $l$ $=0,1$, and 2 . This provides an estimate of the $\sigma \rightarrow \sigma$ contribution for the current PD model potential. For $\beta$ in

$$
\frac{d \sigma}{d \Omega} \propto 1+\beta P_{2}(\cos \theta),
$$

we obtain 1.30 compared to the experimental value of 1.4. The present theoretical value would be expected to be too small since it ignores any $\sigma \rightarrow \pi$ contributions.

\section{ACKNOWLEDGMENTS}

The author wishes to thank I. I. Fabrikant and P. D. Burrow for very useful discussions.

\section{APPENDIX: MEIXNER'S TREATMENT OF EQ. (3)}

As noted in Sec. I, Flammer [10] has given a brief review of the history of treatments of the "angular" and "radial" equations arising out of ellipsoidal coordinates. As far as this author is aware, the only discussions in the literature of the positive-energy solutions to Eq. (3) are in the context of solutions to the time-independent classical wave equation,

$$
\nabla^{2} \psi+k^{2} \psi=0
$$

In this special case, it develops that the two ellipsoidal differential equations are the same, the only difference being the range of the variable appropriate to the $\eta$ and $\xi$ spaces. Many of the previous discussions utilize this, and can write the $S(\xi)$ solution as a simple transform of the $T(\eta)$ solution. This has the unfortunate result of producing an expansion form for $S(\xi)$ inappropriate for our case. The difficulty arises because the relation between the separation constants $\lambda$ and $\epsilon$ is not right for the rather special expansion possible when solving Eq. (A1).

Meixner [9] makes an alternative approach. Flammer gives references to Meixner's work but does not point out that the latter uses a different expansion for the two equations independently and then later shows that in the special case of Eq. (A1) they become equal. Not surprisingly, they are more difficult but Meixner's formulation would be appropriate for our need, a treatment of Eq. (3) for arbitrary real $\lambda$ values. We give here a very brief outline of the treatment.

In the $m=0$ case it starts with a double infinite sum

$$
S(\zeta)=\sum_{k=-\infty}^{\infty} b(\nu)_{k} j_{\nu+k}(\zeta),
$$

where the $j_{\nu+k}(\zeta)$ are generalized spherical Bessel functions, $\zeta=\sqrt{\epsilon} \xi$, and $\nu$ may be complex for our PD potential. This form is different from that usually used when Eq. (A1) is treated directly. It is a two-sided infinite series, and the orders of the spherical Bessel functions are not integers.

If Eq. (A2) is substituted into Eq. (3), a second-order difference equation may be derived for the $b(\nu)_{k}$ coefficients,

$$
q_{k} b(\nu)_{\nu+k}=p_{k} b(\nu)_{\nu+k+2}+r_{k} b(\nu)_{\nu+k-2},
$$

where

$$
\begin{aligned}
& p_{k}=\epsilon \frac{(\nu+k+2)(\nu+k+5)}{(2 \nu+2 k+5)(2 \nu+2 k+3)}, \\
& r_{k}=\epsilon \frac{(\nu+k-4)(\nu+k-1)}{(2 \nu+2 k-1)(2 \nu+2 k-3)},
\end{aligned}
$$

and

$$
\begin{aligned}
q_{k}= & (\nu+k)(\nu+k+1)-(\lambda-\epsilon) \\
& -2 \epsilon \frac{2(\nu+k)^{2}+2(\nu+k)-1}{(2 \nu+2 k-1)(2 \nu+k+3)} .
\end{aligned}
$$

Meixner shows that Eq. (A3) has a convergent solution such that $\left|b(\nu)_{k}\right| \rightarrow 0$ as $k \rightarrow \pm \infty$ so long as $\nu$ has the correct relation to $\lambda$, i.e., $\nu$ is sort of an eigenvalue of the difference equation. We note that, in the set of equations, Eq. (A3) collapses to one term when $\epsilon=0$. When this happens because $E=0$, it shows that an "infinite-wave-length electron" cannot distinguish between a point dipole and a physical dipole of the same moment. At this point $S(\zeta)$ in Eq. (A2) collapses to one term and $\nu$ attains the value predicted from a point dipole. This collapse here is, of course, related to that giving Eq. (26).

When the doubly convergent solution of Eq. (A3) is combined with the spherical Bessel functions, Meixner shows that $S(\zeta)$ of Eq. (A2) is only convergent for $\zeta>\sqrt{\epsilon}$, i.e., $\xi>1$, and is quite slowly convergent close to this. Thus Meixner's basic form is in general contaminated with the logarithmically singular solution at $\xi=1$. This would require determining two independent solutions to eliminate the singularity. In addition, pilot calculations indicate that Meixner's treatment is subject to numerical difficulties when using normal precisions.

All of this may be contrasted with the direct numerical integration of Eq. (3). As stated in Sec. IV, the integration is very stable at normal precisions and requires only real arithmetic. We feel these considerations justify the use of the numerical technique. 
[1] G. Jaffe, Fortschr. Phys. 87535 (1934)

[2] W. B. Baber and H. R. Hasse, Proc. Cambridge Philos. Soc. 31, 564 (1935).

[3] D. R. Bates, K. Ledsham, and A. L. Stewart, Philos. Trans. R. Soc. London, Ser. A 246, 215 (1953).

[4] I. V. Komorov, L. I. Ponomarev, and S. Yu. Slavyarov, Spheroidal and Coulomb Spheroidal Functions (Nauka, Moscow, 1976), in Russian.

[5] K. Takayanagi and Y. Itikawa, J. Phys. Soc. Jpn. 24, 160 (1968).

[6] R. W. Wallis, R. Herman, and H. W. Milnes, J. Mol. Spectrosc. 4, 51 (1960).

[7] M. Abramowitz and I. A. Stegun, Handbook of Mathematical Functions, National Bureau of Standards (U.S. Government Printing Office, Washington, DC, 1970).

[8] L. Y. Wilson and G. A. Gallup, J. Chem. Phys. 45, 586 (1966).

[9] J. Meixner, National Advisory Committee for Aeronautics Technical Memorandum No. 1224, 1944 (unpublished).

[10] C. Flammer, Spheroidal Wave Functions (Stanford University Press, Stanford, California, 1957).

[11] J. Stör and R. Bulirsch, Introduction to Numerical Analysis, 2nd ed. (Springer-Verlag, New York, 1992).

[12] P. M. Morse and H. Feshbach, Methods of Theoretical Physics (McGraw-Hill Book Co., New York, 1953).

[13] T. F. O’Malley, Phys. Rev. 137, A1668 (1965).

[14] It is of interest to note that, at room temperature, an average distance between molecules of $8 \mu \mathrm{m}$ corresponds to a pres- sure of $6 \times 10^{-8}$ torr. It is well known that, physically, longrange multipole potentials are damped out at distances comparable to the average intermolecular distance. Thus our calculations are carried past any such point in typical experiments. When considering real experimental data, effects of molecular rotation will be more dramatic than this damping effect.

[15] W. H. Press, S. A. Teukolsky, W. T. Vetterling, and B. P. Flannery, Numerical Recipes, 2nd ed. (Cambridge University Press, Cambridge, 1992).

[16] I. I. Fabrikant, Sov. Phys. JETP 46, 693 (1977).

[17] I. I. Fabrikant, J. Phys. B 11, 3621 (1978).

[18] J. H. Hendricks, S. A. Lyapustina, H. L. le Clercq, J. T. Snodgrass, and K. H. Bowen, J. Chem. Phys. 104, 7788 (1996).

[19] J. Schiedt, R. Weinkauf, D. M. Neumark, and E. W. Schlag, Chem. Phys. 239, 511 (1998).

[20] M. W. Schmidt, K. K. Baldridge, J. A. Boatz, S. T. Elbert, M. S. Gordon, J. H. Jensen, S. Koseki, N. Matsunaga, K. A. Nguyen, S. J. Su, T. L. Windus, M. Dupuis, and J. A. Montgomery, J. Comput. Chem. 14, 1347 (1993).

[21] C. Desfrançois, H. Abdoul-Carime, and J.-P. Schermann, Int. J. Mod. Phys. B 10, 1339 (1996), a review with many references.

[22] P. D. Burrow, G. A. Gallup, A. M. Scheer, S. Denifl, S. Ptasinska, T. Mark, and P. Scheier, J. Chem. Phys. 124, 124310 (2006). 